\title{
Unique Combinations of epigenetic modifiers synergistically impair the viability of the U87 glioblastoma cell line while exhibiting minor or moderate effects on normal stem cell growth
}

Arshak R Alexanian ( $\nabla$ aalexan@cellrtherapeutics.com )

Cell Reprogramming \& Therapeutics LLC https://orcid.org/0000-0001-6107-9427

Avonlea Brannon

Cell Reprogramming \& Therapeutics LLC

\section{Short Report}

Keywords: Epigenetics, histone covalent modifiers, glioblastoma cells, synergistic effect

Posted Date: November 23rd, 2021

DOI: https://doi.org/10.21203/rs.3.rs-1072015/v1

License: (a) (1) This work is licensed under a Creative Commons Attribution 4.0 International License. Read Full License 


\section{Abstract}

Discoveries made over the last decade have shown that critical changes in cancer cells, such as activation of oncogenes and silencing of tumor suppressor genes are caused not only by genetic but also by epigenetic mechanisms. While epigenetic alterations are somatically heritable, in contrast to genetic changes, they are potentially reversible, making them perfect targets for therapeutic intervention. Covalent modifications of chromatin, such as methylation of DNA and acetylation and methylation of histones, are important components of epigenetic machinery. Multiple recent studies have shown that epigenetic modifiers are candidates for potent new drugs in multiple cancers' therapies, including gliomas, and several clinical trials are ongoing. However, as with other chemotherapeutic drugs, toxicity is one of the main concerns with some of the potent epigenetic drugs. Synergistic combinations of these agents are one approach to overcoming toxicity issues while enhancing efficacy. In this study we demonstrated that while individually BIX01294, an inhibitor of histone methyltransferase G9a, DZNep, an inhibitor of lysine methyltransferase EZH2, and Trichostatin A (TSA), an inhibitor of histone deacetylase at their low concentrations showed a moderate effect on the viability of U87 glioblastoma cells, in combinations they exhibited a synergistic effect. Importantly, these combinations exhibited minimal effect on adipose mesenchymal stem cells (AD-MSCs) growth. Thus, unique combinations and concentrations of epigenetic modifiers, that synergistically attenuated the U87 glioblastoma cells while exhibiting minor or moderate effects on normal stem cell growth, have been discovered.

\section{Introduction}

Epigenetic abnormalities have been well established in cancer cells [1-4]. Epigenetic aberrations, like genetic mutations, can lead to uncontrolled cell division and tumor formation. The most frequently occurring epigenetic changes in cancer cells include changes in histone covalent modifications and DNA methylation. The most common DNA methylation changes in cancer cells are global DNA hypomethylation and gene promoter-specific hypermethylation.

Hypermethylation of gene promoter-specific regions (CpG islands) in cancer cells often initiates inactivation of tumor suppressor genes due to recruitment methyl CpG-binding proteins and their associated chromatin remodeling complexes that in turn inhibit binding of specific transcription factors [5-7]. Hypomethylation of these specific regions of genome largely affects the repeat sequences and transposable elements of DNA, which in turn promote chromosomal instability and an increase in mutation events $[6,8]$. Thus, enzymes such as DNA methyltransferases (DNMT) and methylases that are involved in the regulation of DNA methylation can be good targets for cancer therapy. $[9,10]$.

As mentioned above, histone covalent modifications are another frequently occurring epigenetic alteration in cancer cells. These alterations affect gene transcription via recruitment of chromatin remodeling protein complexes and localized loosening of the nucleosome structure [11]. Levels of different type of histone covalent modifications are maintained by the balance between the activities of 
histone-modifying enzymes that add or remove specific modifications and are thus involved in the regulation of chromatin structure and function.

Malignant cells are characterized by imbalance between activities of these enzymes such as a global reduction in levels of histone acetylation, significant dysregulation of histone methyltransferases (HMTs) and histone demethylases, and by overexpression of histone deacetylases (HDACs) [1, 12-16].

Therefore, in the past decade, several inhibitors of HDACs and HMTs have been extensively investigated in the clinical setting as potential therapies for cancer $[17,18]$.

In our previous studies, we investigated the effect of different epigenetic modifiers and their combinations on the proliferation and viability of normal adult stem cells, such as human mesenchymal stem cells (hMSCs), and malignant glioma cells, such as D54 cells [19]. In these studies, hMSCs or D54 cells were exposed to either different concentrations of specific modulators of chromatin modifying enzymes, such as inhibitors of HMTs, DNMTs, HDACs, or to combinations of these inhibitors. The following four inhibitors were used: 1) BIX01294, inhibitor of HMT G9a and G9a-like protein, which catalyzes methylation of lysine 9 on histone H3; 2) DZNep, inhibitor of lysine methyltransferase EZH2 (KMT6), which is part of the polycomb repressive complex 2 that catalyzes trimethylation of lysine 27 on histone H3; 3) Trichostatin A (TSA), inhibitor of HDACs; and 4) RG108, inhibitor of DNMTs. These epigenetic modifiers were chosen based on several studies demonstrating their involvement in regulation of normal and cancer stem cell proliferation, fate determination, and differentiation.

Initially, three different concentrations for each inhibitor, conditionally called low, medium, and high, were tested for their effect on the viability of hMSCs and D54 cells. Accordingly, the following concentrations had been used: for DZNep 100nM, 500nM and 1uM; for RG-108 50uM, 100uM and 500uM; for BIX01294 $100 \mathrm{nM}, 1 \mathrm{uM}$ and $2 \mathrm{uM}$; and for TSA $20 \mathrm{nM} 100 \mathrm{nM}$ and $200 \mathrm{nM}$. The aim was to identify concentrations that would have minimal effect on normal stem cell growth, while destroying cancer cells. The studies revealed that BIX01294 and TSA in their medium concentrations exhibited a minimal effect on hMSC proliferation while significantly affecting the viability of D54 cells. DZNep showed a similar effect on the viability of D54 cells and hMSCs, and RG-108 showed no effect on both cell types. Next, different combinations of low and medium concentrations of these agents (low for DZNep - 100nM, and RG100 50uM, and medium for BIX01294 - 1uM, and TSA - 100nM) were studied for their effect on D54 and hMSC growth. Results demonstrated that most combinations showed significant effect on D54 cell viability. Since these combinations also affected MSCs proliferation, the medium concentration of BIX01294 and TSA were lowered. These experiments revealed that specific combinations of these agents at their low concentrations significantly affected the viability of D54 cells but exerted a minimal effect on normal stem cell proliferation.

\section{Results}

In this study we aimed to investigate, whether the specific combinations of HMTs and HDACs, that had demonstrated synergistic activity on D54 cell would exhibit a similar effect on another glioma cell line 
such as U87. For cell viability assay the MTT test and trypan blue cell count approach have been used.

Results showed that while individually the DZNep, TSA and BIX01294 at their low concentrations showed a moderate effect on the viability of U87 cells (Fig. 1.a), in combinations they exhibited a synergistic effect (Fig. 1.b), as demonstrated for D54 cells [19]. However, these combinations exhibited minimal or moderate effect on AD-MSCs growth as previously demonstrated for BM-MSCs [19].

TSA and BIX01294 individually at their medium concentrations had a significant effect on U87 cell growth and interestingly increased the proliferation of adipose derived hMSCs (AD-MSCs) (Fig. 2.a). The combination of medium concentrations of TSA and BIX01294 almost completely killed the U87 cells while having a slight effect on AD-MSCs growth (Fig. 2.b). Cells count by trypan blue showed that only 2$3 \%$ of cells that treated with TSA and BIX01294 were survived. No significant differences between low and medium concentrations of DZNep +BIX01294 and DZNep + TSA on U87 cell growth had been detected.

These data, indicate that appropriate combinations and concentrations of epigenetic modifiers, that synergistically impair the viability of two different types of glioblastoma cells (D54 and U87), exhibiting minor or moderate effects on normal stem cell (BM-MSCs and AD-MSCs) growth.

\section{Materials And Methods}

Expansion of U87. U87 glioma cell line was expanded in a-MEM (Gibco, Grand Island, NY, USA) supplemented with 2mM (1\%) GlutaMAX (Gibco, Grand Island, NY, USA), 10\% FBS (R\&D Systems, Minneapolis, MN, USA) and 1\% Pen/Strep (Gibco, Grand Island, NY, USA).

Expansion of MSCs. Fat tissue was obtained from the Medical College of Wisconsin's Tissue Bank (patient remaining anonymous). For purification of MSCs from pre-adipocytes and other cell types, fat tissue was digested in a 1\% Collagenase (Gibco, Grand Island, NY, USA) solution for $1 \mathrm{~h}$ then centrifuged at $50 \mathrm{~g}$ for $1 \mathrm{~min}$. The pellet containing AD-MSCs was washed three times in 1x PBS. These cells were then plated in $25-\mathrm{cm}^{2}$ culture flasks in a-MEM supplemented with $10 \% \mathrm{FBS}, 1 \%$ Pen/Strep, and $1 \%$ GlutaMAX and incubated at $37^{\circ} \mathrm{C}$ in $5 \% \mathrm{CO}_{2}$. The medium was changed every 4 days until the cells reached confluence.

Treatments with chromatin modifying agents.

Cells were seeded in 96 and 6 well plastic plates at a density of $5000 / \mathrm{cm}^{2}$. After $4 \mathrm{~h}$ of attachment, cells were exposed to combinations of three different concentrations of three epigenetic modifiers. The following concentrations were used for each modulator in low and medium doses: BIX01294 - 100nM and 1 UM (Cayman Chemicals, Ann Arbor, MI, USA); DZNep - 100nM, and 500nM (Cayman Chemicals, Ann Arbor, MI, USA); Trichostatin A (TSA) - 20nM, 100nM (Cayman Chemicals, Ann Arbor, MI, USA). 
Cell viability assay with MTT. Cells grown in 96-well-plates for $72 \mathrm{~h}$ were tested for viability with the MTT assay as described previously (35). Briefly, MTT tetrazolium salt (5 mg/mL) (Invitrogen, Eugene, OR, USA) was added to each well, and incubated for $2 \mathrm{~h}$ at $37^{\circ} \mathrm{C}$. The formazan crystals resulting from mitochondrial enzymatic activity on the MTT substrate were solubilized with 12mM SDS (Liberty Scientific, Lisle, IL, USA). Absorbance was measured at $570 \mathrm{~nm}$ using a microplate reader (Accuris Instruments, Edison, NJ, USA). Cell survival was expressed as absorbance relative to that of untreated controls.

Cell Count. For cell count, cells grown in six-well plates for $72 \mathrm{~h}$ were collected and counted using trypan blue stain. Control and each treatment was carried out in triplicate.

\section{Statistical Analysis.}

All data was expressed as mean \pm SEM for the number $(n)$ of independent experiments performed. Differences among the means for all experiments described were analyzed using one-way analysis of variance. Newman-Keul's post hoc analysis was employed when differences were observed by analysis of variance testing $(p<0.05)$.

\section{Discussion}

Aberrant gene expression is a discriminative feature of various types of malignancies, and it is now obvious that epigenetic changes, like genetic alterations, are important in establishing abnormal gene expression patterns. However, epigenetic modifications in contrast to genetic alterations are reversible. Because of these discoveries, the protein complexes that catalyze these modifications have become valuable targets for therapeutic interventions. As a result, a new therapeutic approach, called epigenetic therapy, has been developed in which drugs that can modify chromatin structure and function or DNA methylation are used alone or in combination to affect therapeutic outcomes. During the last decade multiple research studies and clinical trials are focused on the development of combinations of drugs targeting histone and DNA covalent modifications in an attempt to identify synergistic combinations that might enhance antitumor efficacy in the clinic with decreased toxicity. Several recent studies are also focused on the development of drugs and their combinations which exhibit no effect or minimal effect on normal stem cells growth but effectively obliterates cancer cells.

In our previous study we showed that histone methylation and acetylation inhibitors that in relatively small concentrations suppress the growth of D54 cells but exhibited minimal effect on hMSCs proliferation. Most importantly, specific combinations of these histone modifiers selectively killed D54 cells having only little effect on normal stem cells.

Based on these discoveries we decided to test whether the same combination of epigenetic modifiers can exhibit similar effect on another well-known glioma cell line such as U87. Results showed that while individually the DZNep, TSA and BIX01294 at their low concentrations showed a moderate effect on the viability of U87 cells, in combinations they exhibited a synergistic effect, as demonstrated for D54 cells 
[19]. Importantly, these combinations exhibited minimal or moderate effect on AD-MSCs growth, as demonstrated previously for BM-MSCs [19]. These results also showed that the most effective combination was the medium concentrations of TSA + BIX01295 that almost completely killed U87 cells that demonstrated with MTT test and cell count by trypan blue.

Thus, unique combinations and concentrations of epigenetic modifiers, that synergistically attenuated the viability of two different types of glioblastoma cells (D54 and U87) while exhibiting minor or moderate effects on normal stem cell (BM-MSCs and AD-MSCs) growth, have been discovered.

The goal of our current studies is to investigate whether these specific combinations of HMT and HDAC inhibitors that significantly attenuated the viability of D54 and U87 cell lines will exhibit similar effects on other well-established glioma cell lines as well as on glioblastoma cell lines directly derived from patients.

\section{Declarations}

Contributions: Dr Alexanian contributed to study motivation, experimental design and writing the paper. Brannon contribution - technical support.

\section{Data Availability Statements: The datasets generated during and/or analysed during the current study are available from the corresponding author on reasonable request.}

Acknowledgments: This work was supported by Cell Reprogramming \& Therapeutics LLC.

\section{Statements and Declarations}

There are no relevant financial or non-financial competing interests to report.

\section{References}

1. Jones P A and Baylin S B. The epigenomics of cancer. Cell. 2007;128:683-92. 10.1016/j.cell.2007.01.029

2. Esteller M. Cancer epigenomics: DNA methylomes and histone-modification maps. Nat Rev Genet. 2007;8:286-98. 10.1038/nrg2005 
3. Maleszewska M and Kaminska B. Is glioblastoma an epigenetic malignancy? Cancers (Basel). 2013;5:1120-39. 10.3390/cancers5031120

4. Yu D H, Waterland R A, Zhang P, Schady D, Chen M H, Guan Y, et al. Targeted p16(Ink4a) epimutation causes tumorigenesis and reduces survival in mice. J Clin Invest. 2014;124:3708-12. $10.1172 /$ jci76507

5. Sasaki $\mathrm{H}$ and Matsui Y. Epigenetic events in mammalian germ-cell development: reprogramming and beyond. Nat Rev Genet. 2008;9:129-40. 10.1038/nrg2295

6. Kulis M and Esteller M. DNA methylation and cancer. Adv Genet. 2010;70:27-56. 10.1016/b978-0-12380866-0.60002-2

7. Feinberg A. DNA methylation in cancer: three decades of discovery. Genome Med. 2014;6:36. $10.1186 /$ gm553

8. Wilson A S, Power B E and Molloy P L. DNA hypomethylation and human diseases. Biochim Biophys Acta. 2007;1775:138-62. 10.1016/j.bbcan.2006.08.007

9. Brueckner B, Kuck D and Lyko F. DNA methyltransferase inhibitors for cancer therapy. Cancer J. 2007;13:17-22. 10.1097/PPO.0b013e31803c7245

10. Gnyszka A, Jastrzebski Z and Flis S. DNA methyltransferase inhibitors and their emerging role in epigenetic therapy of cancer. Anticancer Res. 2013;33:2989-96.

11. Strahl B D and Allis C D. The language of covalent histone modifications. Nature. 2000;403:41-5. $10.1038 / 47412$

12. Sharma S, Kelly T K and Jones P A. Epigenetics in cancer. Carcinogenesis. 2010;31:27-36. 10.1093/carcin/bgp220

13. Shi Y. Histone lysine demethylases: emerging roles in development, physiology and disease. Nat Rev Genet. 2007;8:829-33. 10.1038/nrg2218

14. Yoo C B and Jones P A. Epigenetic therapy of cancer: past, present and future. Nat Rev Drug Discov. 2006;5:37-50. 10.1038/nrd1930

15. Nakagawa M, Oda Y, Eguchi T, Aishima S, Yao T, Hosoi F, et al. Expression profile of class I histone deacetylases in human cancer tissues. Oncol Rep. 2007;18:769-74.

16. Yang H, Salz T, Zajac-Kaye M, Liao D, Huang S and Qiu Y. Overexpression of histone deacetylases in cancer cells is controlled by interplay of transcription factors and epigenetic modulators. FASEB J. 2014;28:4265-79. 10.1096/fj.14-250654

17. Lane A A and Chabner B A. Histone deacetylase inhibitors in cancer therapy. J Clin Oncol. 2009;27:5459-68. 10.1200/jco.2009.22.1291

18. Spannhoff A, Sippl W and Jung M. Cancer treatment of the future: inhibitors of histone methyltransferases. Int J Biochem Cell Biol. 2009;41:4-11. 10.1016/j.biocel.2008.07.024

19. Alexanian A R and Huang Y W. Specific combinations of the chromatin-modifying enzyme modulators significantly attenuate glioblastoma cell proliferation and viability while exerting minimal effect on normal adult stem cells growth. Tumour Biol. 2015;10.1007/s13277-015-3654-1 
Figures
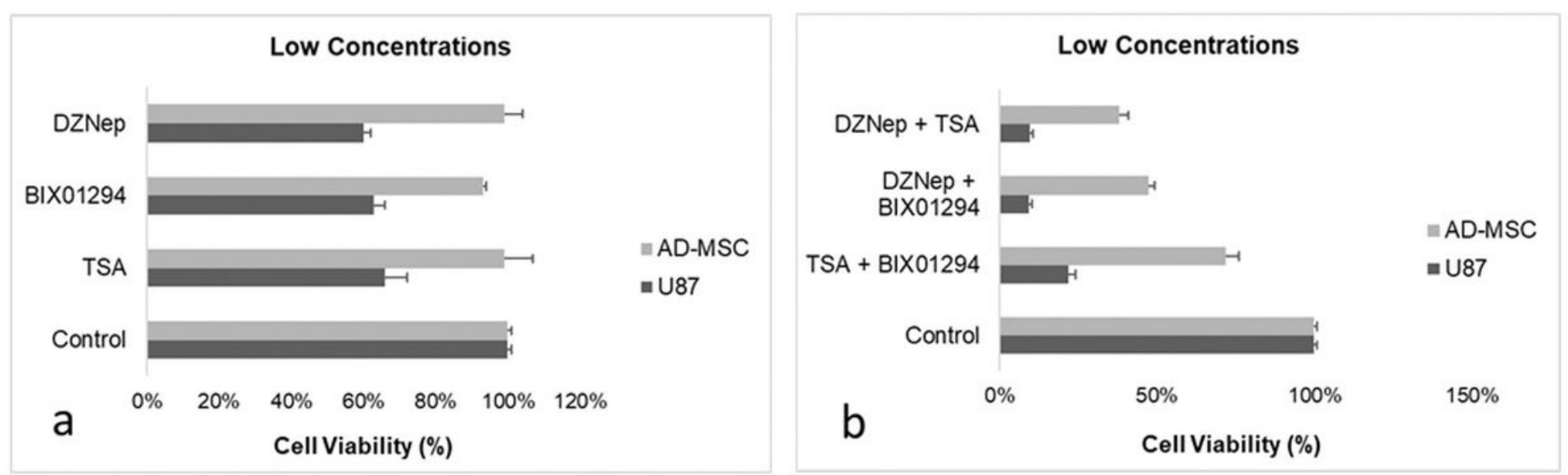

\section{Figure 1}

MTT cell viability assay for U87 cells and human adipose-derived hMSCs treated with low concentrations of epigenetic modifiers DZNep, BIX01294, and TSA individually (a) and in combinations (b).
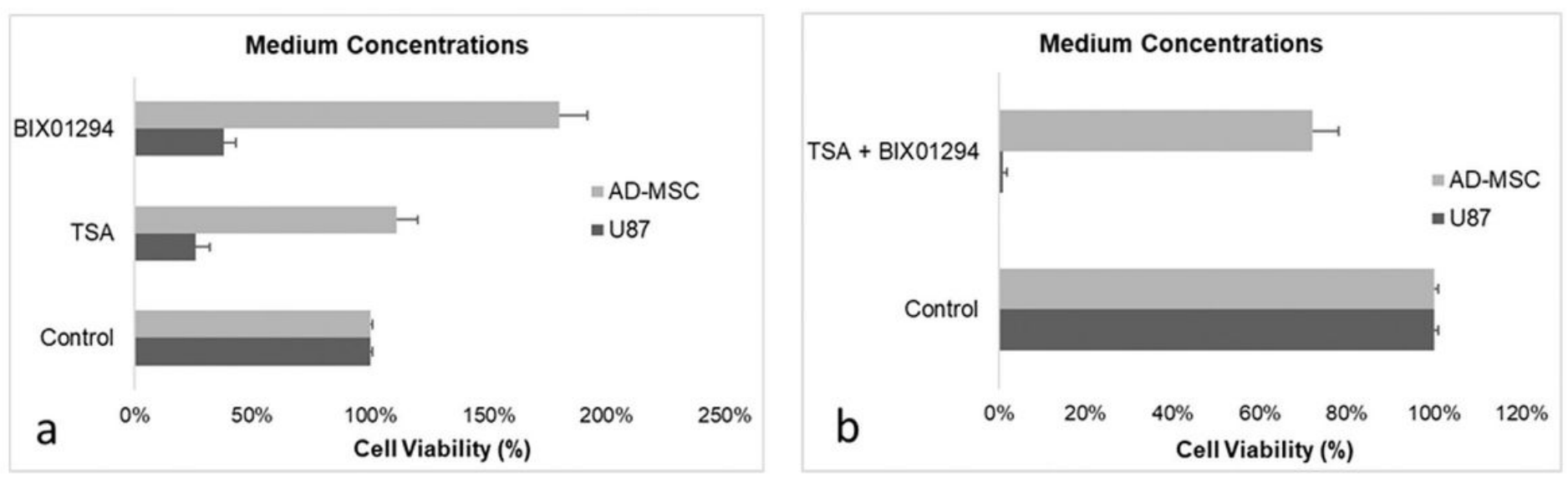

Figure 2

Cell Viability Assay for U87 cells and hMSCs treated with medium concentrations of epigenetic modifiers BIX01294 and TSA individually (a) and in combination (b). 